\title{
Coordination under global random interaction and local imitation
}

Citation for published version (APA):

Khan, A. (2013). Coordination under global random interaction and local imitation. Maastricht University, Graduate School of Business and Economics. GSBE Research Memoranda No. 004 https://doi.org/10.26481/umagsb.2013004

Document status and date:

Published: 01/01/2013

DOI:

10.26481/umagsb.2013004

Document Version:

Publisher's PDF, also known as Version of record

\section{Please check the document version of this publication:}

- A submitted manuscript is the version of the article upon submission and before peer-review. There can be important differences between the submitted version and the official published version of record.

People interested in the research are advised to contact the author for the final version of the publication, or visit the DOI to the publisher's website.

- The final author version and the galley proof are versions of the publication after peer review.

- The final published version features the final layout of the paper including the volume, issue and page numbers.

Link to publication

\footnotetext{
General rights rights.

- You may freely distribute the URL identifying the publication in the public portal. please follow below link for the End User Agreement:

www.umlib.nl/taverne-license

Take down policy

If you believe that this document breaches copyright please contact us at:

repository@maastrichtuniversity.nl

providing details and we will investigate your claim.
}

Copyright and moral rights for the publications made accessible in the public portal are retained by the authors and/or other copyright owners and it is a condition of accessing publications that users recognise and abide by the legal requirements associated with these

- Users may download and print one copy of any publication from the public portal for the purpose of private study or research.

- You may not further distribute the material or use it for any profit-making activity or commercial gain

If the publication is distributed under the terms of Article $25 \mathrm{fa}$ of the Dutch Copyright Act, indicated by the "Taverne" license above, 


\section{Maastricht University}

Abhimanyu Khan

Coordination under Global Random I nteraction and Local I mitation

$\mathrm{RM} / 13 / 004$

\section{GSBE}

Maastricht University School of Business and Economics

Graduate School of Business and Economics

P.O Box 616

NL- 6200 MD Maastricht

The Netherlands 


\title{
Coordination under Global Random Interaction and Local Imitation*
}

\author{
Abhimanyu Khan ${ }^{\dagger}$
}

January 30, 2013

\begin{abstract}
We study stochastically stable behaviour in $2 \times 2$ coordination games where the riskdominant equilibrium differs from the Pareto-efficient equilibrium. Individuals are randomly matched to another individual in the population (with full support) and they choose strategies by imitating the most successful individual they observe. So, while individuals interact globally, their observation, as determined by their social network, may be local. In the benchmark model, all individuals observe each other, and hence, an individual imitates the strategy of the most successful individual in the entire population; here, the stochastically stable outcome corresponds to the situation where everyone coordinates on the Pareto-efficient equilibrium. While this outcome is always stochastically stable even when observability is incomplete, the state where everyone plays the action of the risk-dominant equilibrium may be stochastically stable as well. Reasonably tight sufficient conditions for unique stochastic stability of the state where all individuals play the Pareto-efficient equilibrium strategy include each individual observing at least four other individuals or when each individual observes the same number of other individuals.
\end{abstract}

Keywords: evolution of behavior, coordination games, local imitation, stochastic stability. JEL classification codes: C73, D03.

${ }^{*}$ I would like to thank Meenakshi Ghosh, Jean-Jacques Herings, William Sandholm and Rene Saran for their helpful comments and suggestions. I am indebted to Ronald Peeters for his meticulous guidance that has immensely and substantially improved the paper.

${ }^{\dagger}$ Department of Economics, Maastricht University, P.O. Box 616, 6200 MD Maastricht, The Netherlands. Email: a.khan@maastrichtuniversity.nl. 


\section{Introduction}

This paper studies the evolution of behaviour in coordination games when individuals choose their strategy by imitation of the most successful individual they observe. The individual's scope of strategic interaction is global, in the sense that the co-player may be drawn from the entire population. However, due to informational or other constraints, the individual is only locally informed, i.e. he is aware of the experience of only a subset of individuals in the population, namely his neighbours. Individuals imitate the most successful individual in their respective neighbourhood and this may differ from imitating the most successful individual. We investigate the effect of such a "local" imitation protocol when the interaction is in a wider environment.

The set-up has three principal ingredients. Firstly, the strategic nature of the situation, that is recurrent, is described by a $2 \times 2$ coordination game where the Pareto-efficient coordination equilibrium differs from the risk-dominant coordination equilibrium. There is, therefore, a divergence between the preferred equilibrium and the "safer" equilibrium. An individual is randomly matched with another co-player from a finite population. Random matching carries the import that there are circumstances where an individual does not have prior knowledge of the person he will be interacting with. This situation ensues recurrently and so, an individual has to choose a strategy each period.

Secondly, we assume that an individual does not have complete information about the environment that he is a part of. He is able to observe, acquire and exchange information (i.e. strategy choice and payoff received) from a subset of individuals in the entire population, say his neighbours. This is modeled by placing individuals on a network, where the links of an individual determine his social network and his set of neighbours. The links are assumed to be undirected (i.e. the flow of information between two linked individuals is bidirectional) and exogenously given. ${ }^{1}$ Even though individuals may not observe everyone in the population, it is likely that any two individuals in the population are connected either directly or indirectly via other individuals. So, we assume the network to be connected.

Thirdly, we assume that an individual, owing perhaps to reasons of complexity, informational constraints or some form of bounded rationality, imitates the most successful individual (including himself) that he observes in the previous period. Apesteguia et al. (2007) find experimental support for imitative behaviour while Björnerstedt and Weibull (1996) provide

\footnotetext{
${ }^{1}$ The interpretation is that the links are given exogenously by history and social factors and is inherited by an individual. Of course, there may be situations wherein the process of link formation or deletion may merit as much attention as the choice of strategy. For example, Goyal and Vega-Redondo (2005) discuss the case where not only do individuals play a coordination game, but are also able to establish unilateral costly links with others; these links determine the set of people with whom the interaction occurs. However, here, the interest is not how the information structure evolves via the network; rather, the network is treated as a modeling device that determines information percolation.
} 
theoretical support for such behaviour in a game-theoretical context. ${ }^{2}$ In this model, imitation may be interpreted as a means by which an individual tries to grope for the action that might result in a better outcome; and since an individual by himself is not aware of the state of the entire world, he also relies on the experience of his acquaintances.

We ask the following questions: what is the effect of incomplete observation induced local imitation on the stochastically stable outcome when individuals interact globally? When are societies able to coordinate on the Pareto-efficient equilibrium strategy? That is, what are the informational network structures under which a society is able to converge to the state in which all players coordinate on the Pareto-efficient equilibrium?

At this juncture, we present a couple of examples where the described model is relevant. Consider a labour market where individuals gather for employment in each period. Employers randomly choose individuals in pairs. Suppose there are two effort levels (high and low), which are unobservable. Assume a joint-production technology, where the output depends on the minimum effort in a pair (as in a minimum effort game). Effort being unobservable, individuals are paid a variable wage in accordance with the realised output. The utility of an agent is the effort adjusted wage, i.e. the wage received less the disutility of effort. Assume that the wage differential (when both individuals in a pair put in high effort compared to when neither of them do so) justifies the disutility of higher effort. The essence of this situation can be captured by a symmetric coordination game where it is better for both individuals to coordinate on a high effort; but, in case of mis-coordination, the payoff received by exerting lower effort is strictly higher than the returns from higher effort. The following period, they imitate the effort level that had resulted in the highest payoff amongst the individuals that they are are connected with.

A second example involves the evolution of socio-cultural features such as punctuality, which may be modeled as a coordination game (as in Basu and Weibull (2003)). Two randomly selected individuals are supposed to meet at a pre-specified time. Coordinating on punctual behaviour is better for both individuals as opposed to a situation when both of them are impunctual. In case of mis-coordination of actions, the punctual individual incurs a cost (for example, of waiting). Thus, both individuals arriving on time represents the Paretoefficient outcome while both individuals being tardy represents the risk-dominant outcome. An individual selects a particular course of action and receives a payoff depending on his action and that of his randomly selected partner. Individuals exchange their respective experiences with their neighbours and thereafter, an individual selects the strategy that yielded the highest payoff. In either case, our interest lies in the mode of behaviour that is expected to emerge in the long-run. How does the information structure affect the custom that individuals settle on?

\footnotetext{
${ }^{2}$ Imitation of successful behaviour also finds mention in social and biological literature such as Dawkins (1976) and Ulmann-Margalit (1978) as a propellant of human intelligence.
} 
A natural benchmark for the model of imitation under incomplete observability presented here, is imitation under complete observability, i.e. when individuals can observe everyone and hence, imitate the strategy choice of the most successful individual in the entire population. Robson and Vega-Redondo (1996) present a similar model, where in each period, every individual interacts with $v$ other randomly chosen individuals to play a coordination game. The actions and payoffs of individuals are observable to all and the action that yields the highest average payoff is imitated. Here, for tractability, we assume that an individual interacts with only one individual per period, and that individuals imitate the individual with the highest payoff. As the action of a more successful individual is more salient than a strategy that gives the higher average payoff, it may be more plausible to assume that in certain situations, the more successful individual is imitated. ${ }^{3}$

The full observability model is a particular instance of the model of incomplete observability. The latter generates the former when the network is complete, i.e. each individual is linked to every other individual. In Proposition 1, we show that in either case, the defined imitation rule leads to all individuals choosing the same strategy. So, the process converges to either the Pareto-efficient equilibrium or the risk-dominant equilibrium. To obtain the stochastically stable outcome, infinitesimally small perturbations are introduced in the imitation dynamic. These perturbations may be interpreted as the propensity of individuals to choose a strategy not necessarily prescribed by the specified imitation rule, owing perhaps to tendencies to experiment or to make mistakes. This makes the transition from the state where everyone uses the strategy of the Pareto-efficient equilibrium to the state where everyone adopts the risk-dominant equilibrium strategy, and vice versa, possible. The equilibrium that is more resistant to such perturbations is stochastically stable.

In the full observability model, the Pareto-efficient equilibrium is the stochastically stable state (see Proposition 2) for the reason that the risk-dominant equilibrium is relatively more susceptible to experimentation by individuals. To elaborate, suppose that the state is given by all individuals playing the risk-dominant strategy. If two individuals experiment with the strategy of the Pareto-efficient equilibrium, and are also matched to each other (a random event with positive probability), they obtain the highest payoff. This is observed by everyone and their strategy is imitated. The Pareto-efficient equilibrium is more resistant as, for populations that contain at least six individuals, the transition to the risk-dominant equilibrium requires at least three experimentations.

With limited observability (i.e. when the connected network is not complete), while the Pareto-efficient equilibrium is always stochastically stable, it may not be uniquely so; the

\footnotetext{
${ }^{3}$ An observation consistent with this assumption involves people who desire to be actors or sportpersons. In light of the very small percentage of people who find success, the average payoff for choosing such an action might not be very high. It seems that the attractiveness of such professions is (at least partially) driven by the salience of people who actually end up being successful.
} 
risk-dominant equilibrium may be stochastically stable as well. The reason for the stability of the Pareto-efficient equilibrium is similar. If two individuals experiment to adopt the strategy of the Pareto-efficient equilibrium when all else play the risk-dominant strategy, and if these two individuals are also matched, they obtain the highest payoff. This is observed by their neighbours, who then imitate this strategy. With positive probability, the strategy spreads to the neighbours' neighbours and finally, because of connectedness of the network, to the entire population in finite time. The reverse transition takes at least two experimentations. (This establishes the stochastic stability of the Pareto-efficient equilibrium and this is the content of Proposition 3.) With an example, we show that the reverse transition may be possible with two experimentations. This possibility arises precisely because of limited observability; the risk-dominant equilibrium strategy may spread by imitation because it is the most successful strategy that individuals observe. Hence, this example illustrates that limited observability does make a difference.

A natural follow-up question concerns conditions under which the Pareto-efficient equilibrium is expected to uniquely prevail and, to this end, we identify two alternative (sufficient) conditions. One is that each individual, independent of the size of the population or the payoff structure of the game, needs to observe (i.e. be linked to) at least four other individuals (Proposition 4). This can be interpreted as an absolute criterion; a minimum level of observability that, independent of other factors, suffices to support the unique stability of the Pareto-efficient equilibrium. Proposition 5 reveals a second condition that deals with regular networks; the Pareto-efficient equilibrium is uniquely stable when the network is regular (i.e. all individuals are linked to the same number of individuals). Further, we present examples to show that the sufficient conditions mentioned above are not very slack.

Related papers which posit an imitation dynamic in a game-theoretic setting (as opposed to imitation in a decision-theoretic framework as in Ellison and Fudenberg $(1993,1995)$ or Banerjee and Fudenberg (2004)) include Robson and Vega-Redondo (1996) and Eshel et al. (1998). The former assumes that an individual interacts with randomly chosen individuals from the entire population and imitates the strategy that gives the highest average payoff in the entire population. The latter focusses on local structures: not only does an individual interact only with his neighbours, but imitates the strategy that yields the highest average payoff in his neighbourhood. However, irrespective of the scope being global or local, the interaction and information spheres coincide in these papers. Alós-Ferrer and Weidenholzer (2008) make a distinction between these two by assuming that the information sphere is larger than the interaction sphere: while individuals play the coordination game in their neighbourhood, they hear of the behaviour of a larger set of individuals; individuals imitate the most successful individual they know of and this may be an individual they never interact with themselves (adoption of best-practises in an industry is given as an example). The 
motivation here is different: individuals might realistically not be able to observe everyone in the population, but the person with whom they interact might be randomly chosen. Given that they cannot observe everyone, they imitate the most successful individual they can observe. This results in a situation where the interaction sphere is larger than the information sphere.

The structure of the paper is as follows. Section 2 presents the model and preliminaries. The results are contained in Section 3. Section 4 concludes. Some proofs are presented in the appendix.

\section{$2 \quad$ Model and Preliminaries}

We consider a finite population comprising of $M$ individuals and denote the set of all individuals by $I$. In each period, an individual chooses between strategies $P$ and $R$, after which he is randomly matched to another individual; there is a strictly positive probability of every possible match and it is possible to incorporate the feature that, based on certain factors, some matches are more probable than others. Throughout the paper, it is assumed that the population is made up of an even number of individuals (to avoid the situation where someone is left unmatched) and contains at least six individuals; so, $M \geq 6$ and even. ${ }^{4}$ The paired individuals interact in a $2 \times 2$ symmetric coordination game. The payoff that an individual receives on choosing strategy $s_{i}$ is denoted by $u_{i}\left(s_{i}\right)$ (suppressing the dependence of the payoff on the co-player's strategy) and it is in accordance with the matrix below.

\begin{tabular}{c|cc|}
\multicolumn{1}{c}{} & \multicolumn{1}{c}{$P$} & \multicolumn{1}{c}{$R$} \\
\cline { 2 - 3 }$P$ & $a, a$ & $b, c$ \\
$R$ & $c, b$ & $d, d$ \\
\cline { 2 - 3 } & &
\end{tabular}

We assume that (1) $a>c, d>b,(2) a>d$ and (3) $a+b<c+d$. Condition (1) says there are two strict equilibria represented by the strategy pairs $(P, P)$ and $(R, R) ;(2)$ says the first equilibrium payoff dominates the other, while (3) says that the latter is risk dominant.

After an individual receives the appropriate payoff, he observes the experience (i.e. chosen strategy and payoff received) of a set of other individuals, called his neighbours. The set of neighbours of an individual $i$, denoted by $N(i)$, is determined by the structure of links in an exogenous network. Individual $i$ is directly connected to all individuals in $N(i)$. It is assumed that $i \notin N(i)$. However, since an individual always knows of his own experience, his reference set is $N(i) \cup\{i\}$. The links are undirected so that if individual $i$ observes individual $j$, then individual $j$ also observes individual $i$. Thus, $j \in N(i)$ implies $i \in N(j)$. Let $N^{2}(i)$ represent the individuals who are neighbours or neighbours of the neighbours of

\footnotetext{
${ }^{4}$ Under these conditions, with full observability, the state where everyone plays the strategy of Paretoefficient equilibrium is stochastically stable. See footnote 6 for more details.
} 
individual $i$, i.e. $N^{2}(i)=\cup_{j \in N(i)} N(j) \cup N(i) \backslash\{i\}$. Further, $N^{k}(i)$ is defined recursively: $N^{k}(i)=\cup_{j \in N^{k-1}(i)} N(j) \cup N^{k-1}(i) \backslash\{i\}$ for $k \geq 2$; it represents the set of other individuals who are at most $k$ links away from individual $i$. Since we assume the network to be connected, for any individual $i$, there exists an integer $K$ (for instance, $K=M-1$ ) such that $N^{K}(i)=I \backslash\{i\}$. So, starting from an individual $i$, every other individual is at a distance of at most $K$ links. The network is said to be complete if, for all individuals $i \in I, N(i)=I \backslash\{i\}$; we refer to this as the situation of full observability. Otherwise, we are in the realm of incomplete observability. The network, $N$, is defined by the direct links of the individuals: $N=\{N(i)\}_{i \in I}$. The set of all possible connected networks is denoted by $\mathcal{N}$.

After realising his own payoff and observing the experience of his neighbours, individual $i$ chooses, in the succeeding period, the strategy of the most successful individual he observes. Therefore, he chooses $s_{i}^{*}=\operatorname{argmax} u_{j}\left(s_{j}\right)$, where $j \in N(i) \cup\{i\}{ }^{5}$ When the network is complete, $s_{i}^{*}=s_{j}^{*}$ for any two individuals $i$ and $j$, i.e. everyone imitates the same strategy. On the other hand, when the network is not complete, then the strategy $s_{i}^{*}$ may not coincide with the strategy of the most successful individual in the population. It is also possible that $s_{i}^{*}$ and $s_{j}^{*}$ are different. Hence different individuals may revise to different strategies under limited observability.

The elements above describe the evolution of behaviour in the society. Individuals receive payoffs depending on their strategy and the choice of strategy of the randomly matched partner. For the following period, they revise their strategy simultaneously and choose the strategy of the most successful individual they know of. This situation continues recurrently under the specified dynamics of "local" imitation.

For a given network and specification of the random matching process, the state of the process at any given point in time is described by the strategy choice of each individual and the state space can be represented by $\Omega=\{P, R\}^{M}$. It is then possible to define, for a given network and specification of the random matching process, a Markov process $Q$ over the state space that indicate the probabilities of transition between states. Let $\vec{P}(\vec{R})$ denote the state where all individuals play strategy $P(R)$. In addition, we define a statistic that can be derived from the state of the process: $\omega$, the number of individuals using strategy $P$. For example, when the state is $\vec{P}$, then $\omega=M$ - everyone uses the strategy of the Pareto-efficient equilibrium. The initial state of the process, and thus the initial description of the derived statistic $\omega$, is given arbitrarily.

An absorbing state of the process is defined as a state in which the system remains locked in and there is no possibility of transiting to a different state. Therefore, if the process hits an absorbing state, then the number of individuals who use strategy $P(R)$ does not change. A first observation is that for any network $N \in \mathcal{N}$, the only absorbing states of the process

\footnotetext{
${ }^{5}$ Note that because the payoffs are distinct, for individual $i, s_{i}^{*}$ is unique.
} 
of local imitation are the states $\vec{P}$ and $\vec{R}$, where all individuals choose the same action. That these two states are absorbing is obvious: if everyone uses the same strategy, then there is no other strategy to imitate and hence the process is locked in thereafter. Further, any other state is non-absorbing; from any other state, the process eventually ends up in either $\vec{P}$ or $\vec{R}$. This is the content of the proposition below, the proof of which is in the appendix.

Proposition 1. For any connected network $N \in \mathcal{N}$, the absorbing states of the unperturbed process of local imitation are $\vec{P}$ and $\vec{R}$.

This proposition is immediate for a complete network i.e. under full observability. Everyone imitates the action of the most successful individual, and since payoffs are distinct, all individuals revise to the same strategy, thereby leading the process to one of the absorbing states, $\vec{P}$ or $\vec{R}$. However, when the network is not complete (i.e. under incomplete observability), it is possible that the random matching unfolds in a way such that $s_{i}^{*}=P$ for individual $i$ whereas $s_{j}^{*}=R$ for another individual $j$. Individuals $i$ and $j$ would then imitate different strategies and the transition to the absorbing states would not be immediate; we show this with the help of the following example.

Example. In Figure 1 below, the solid lines in the network of ten individuals indicate the links. In the initial state, the individuals numbered one to five use strategy $P$, while the individuals numbered six to ten choose strategy, $R$. The dotted lines indicate a particular realisation of the random matching in a period. The triplet alongside each individual indicates, from left to right, his current strategy, payoff and strategy he will imitate next period. For example, individual 5 plays strategy $P$ and receives a payoff of $b$; in the next period he continues to play strategy $P$. With the indicated matching, it is easily verified that all individuals hold

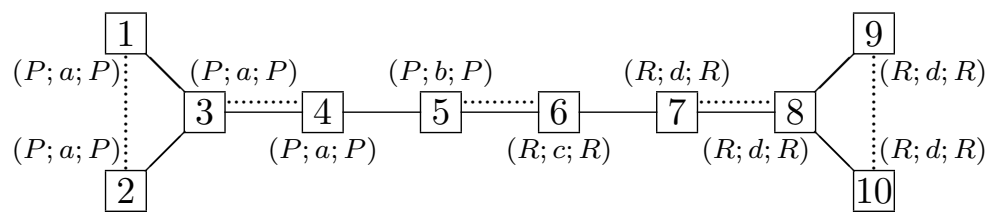

Figure 1: An example.

on to their current strategy. The first four (last four) individuals only observe individuals with strategy $P(R)$, and hence do not change their strategy. Individual 5 receives a payoff of $b$ with strategy $P$ and in addition, he observes individual 4 and individual 6 receiving a payoff of $a$ and $c$ with strategies $P$ and $R$ respectively; for him strategy $P$ is the more successful strategy (even though he himself does not fare well with that strategy) and he imitates it in the next period. For similar reasons, individual 6 chooses strategy $R$ in the next period.

Thus, in this case, unlike in the full observability model, after a period of strategy revision, the population may consist of both strategy $P$ and strategy $R$ players. However, the transition to 
$\vec{P}$ or $\vec{R}$ happens eventually. Crucial to the argument is that if the state is other than $\vec{P}$ or $\vec{R}$, then because of connectedness of the network there exists at least one individual who observes at least one individual who uses strategy $P$ and at least one individual who uses strategy $R$. Using this, (we show in the proof in the appendix) it is possible to construct a path in which either the number of individuals who use strategy $P$ or the number of individuals who use strategy $R$ increase until all individuals in the population use the same strategy.

The process of pure imitation is now perturbed with the possibility of making mistakes or experimenting; with an independent and time invariant probability $\varepsilon$, an individual plays a strategy randomly. Let $Q_{\varepsilon}$ denote this perturbed Markov process. This process does not have any absorbing states due to the randomness in strategy choice of each individual and it is possible to transit from the previously described absorbing states of the unperturbed process $Q$. Standard results on Markov process indicate that the perturbed process $Q_{\varepsilon}$ has a unique invariant distribution $\mu(\varepsilon)$, i.e. $\mu(\varepsilon) Q_{\varepsilon}=\mu(\varepsilon)$. To determine the long-run outcome, we use the notion of stochastic stability (see Young $(1993,1998)$ and Kandori et al. (1993)), which selects the states that receive a positive weight in the limiting invariant distribution, $\mu^{*}=\lim _{\varepsilon \downarrow 0} \mu(\varepsilon)$. The absorbing state of the unperturbed process that is most resistant in the face of these experimentations is stochastically stable and is expected to be observed more frequently in the long-run, as individuals' probability of making random choices vanishes to zero.

\section{Results}

By Proposition 1, the process reaches one of the absorbing states of the unperturbed process in finite time. Let $\rho(\vec{P} \rightarrow \vec{R})$ and $\rho(\vec{R} \rightarrow \vec{P})$ denote the resistance or minimum number of experimentations required to move from the state $\vec{P}$ to the state $\vec{R}$ and vice-versa respectively. The state $\vec{P}$ is stochastically stable if $\rho(\vec{P} \rightarrow \vec{R}) \geq \rho(\vec{R} \rightarrow \vec{P})$; further, it is uniquely so if the inequality is strict. Similarly, the state $\vec{R}$ is stochastically stable if $\rho(\vec{P} \rightarrow \vec{R}) \leq \rho(\vec{R} \rightarrow \vec{P})$ and uniquely so if the inequality is strict.

First, consider the benchmark model of full observability where the network is complete. We argue (as in Robson and Vega-Redondo (1996)) that $\vec{P}$ is the unique stochastically stable state. The resistance of the transition from $\vec{R}$ to $\vec{P}$ is equal to 2, i.e. $\rho(\vec{R} \rightarrow \vec{P})=2$. Suppose that the initial state is $\vec{R}$. Experimentation to strategy $P$ by one individual only results in a reversion to the initial state; however, if two individuals experiment with strategy $P$, and they are matched together (a positive probability event), then they realise the highest possible payoff of $a$. This is observed by everyone and imitation leads to the state $\vec{P}$. The transition from state $\vec{R}$ to $\vec{P}$, on the other hand, requires strictly more than two experimentations. To see this, note that when any two individuals experiment with strategy $R$ when the state is $\vec{P}$, since there are at least six individuals $(M \geq 6)$, there are still at least two individuals that 
collect a payoff of $a$ by using strategy $P$. So, strategy $P$ is imitated by all individuals and the state reverts to $\vec{P} .{ }^{6}$ This is summarised in the following proposition.

Proposition 2. For a complete network, which means full observability, the state where everyone plays the Pareto-efficient equilibrium strategy is the unique stochastically stable state.

Now, suppose the connected network is not complete, implying that there is at least one individual who does not observe everyone else. Is the state $\vec{P}$ still stochastically stable? The following proposition (the proof is in the appendix) reveals that it indeed is.

Proposition 3. For any connected network $N \in \mathcal{N}$, the state $\vec{P}$ where everyone uses the Pareto-efficient equilibrium strategy $P$ is a stochastically stable state.

Now, because of limited observability, the arguments made earlier to establish Proposition 2 do not apply directly. To illustrate, suppose that starting from the initial state $\vec{R}$, two individuals experiment with strategy $P$. With positive probability, they are matched to each other and they receive the highest payoff possible; but due to limited observability, this may not be observed by all and so, all individuals may not imitate the use of strategy $P$ in the next period. However, the transition from $\vec{R}$ to $\vec{P}$ occurs with positive probability following experimentation by two individuals. Connectedness of the network makes this contagion possible and so, $\rho(\vec{R} \rightarrow \vec{P})=2$. It can also be shown that the transition in the other direction, i.e. from $\vec{P}$ to $\vec{R}$ needs at least two such experimentations; so, $\rho(\vec{P} \rightarrow \vec{R}) \geq 2$. Since $\rho(\vec{P} \rightarrow \vec{R}) \geq \rho(\vec{R} \rightarrow \vec{P})$, the result follows.

The proposition only indicates that the state where everyone plays the Pareto-efficient strategy is stochastically stable; it does not imply that it is the unique stochastically stable state. In fact, the state $\vec{R}$ may be stochastically stable as well. This possibility arises, and is demonstrated in the example below, when the transition from $\vec{P}$ to $\vec{R}$ is effected with two experimentations.

Example. Figure 2 presents a network, for which, we show a sequence of random matching of individuals such that the transition from $\vec{P}$ to $\vec{R}$ is affected by initial experimentations. The solid lines indicate the links in the network of ten individuals. The initial state in Period 0 is $\vec{P}$, as indicated by the strategy choice of each individual in the diagram. Now, suppose that in Period 1, individual 2 and individual 3 experiment with strategy $R$. The realisation of the random match is depicted with dotted lines: for example, individual 1 is matched to individual 2. The triplet alongside each individual indicates, from left to right,

\footnotetext{
${ }^{6}$ If $M=2$, the transition from $\vec{P}$ to $\vec{R}$ is possible with one experimentation; further, the transition in the reverse direction take two experimentations. Thus, for $M=2$, the state $\vec{R}$ is uniquely stochastically stable. If $M=4$, then a minimum of two experimentations are required to induce the transition from $\vec{P}$ to $\vec{R}$ and from $\vec{R}$ to $\vec{P}$; so, both $\vec{P}$ and $\vec{R}$ are stochastically stable states. We assume $M \geq 6$ as it is a more reasonable population size; also, it results in the $\vec{P}$ being the unique stochastically stable state under full observability and makes for meaningful comparisons with the outcomes of incomplete observability.
} 
Period 0

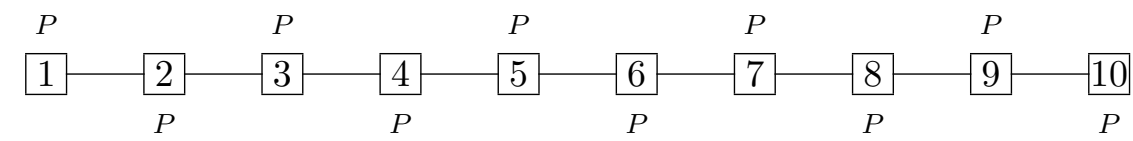

$$
(P ; b ; R) \quad(R ; c ; R) \quad(P ; a ; P) \quad(P ; a ; P) \quad(P ; a ; P)
$$

\section{Period 1 \\ Period 1}

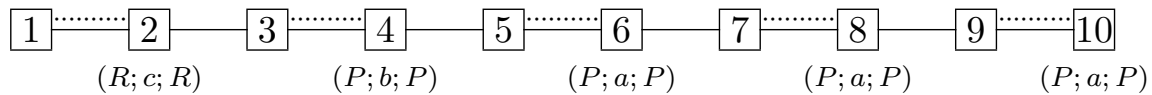

Period 2

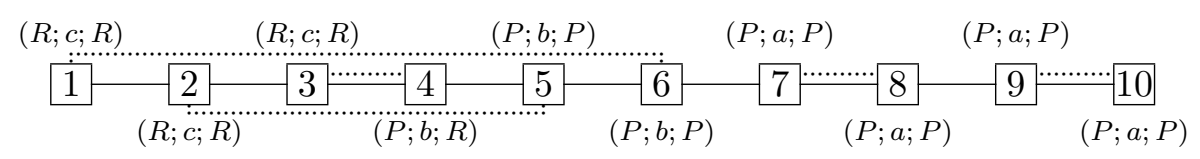

Period 3

$$
(R ; c ; R) \ldots \ldots(R ; c ; R) \ldots
$$

Period 4

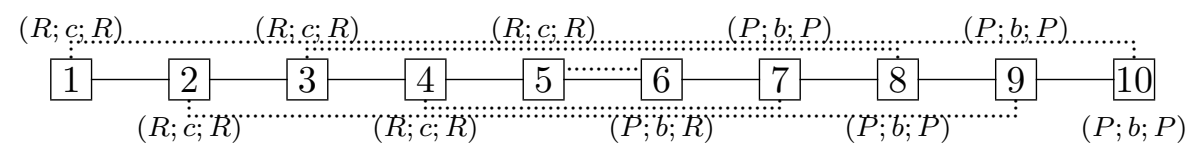

Period 5

$$
(R ; c ; R) \ldots \ldots(R ; c ; R) \text { (R; }
$$

Period 6

$$
(R ; c ; R) \text { (R; }
$$

Period 7

$$
(R ; c ; R) \text { ( }
$$

Period 8

$$
(R ; c ; R) \quad(R ; c) \quad(R ; C ; R)
$$

Figure 2: Example to illustrate the stochastic stability of state $\vec{R}$.

the current strategy, the payoff received and the strategy to be chosen by imitation in the next period. As a result of the realised matching of individuals, for the first three individuals $(i=1,2,3), R$ is the more successful strategy in the respective neighborhoods, while each of the remaining seven individuals $(i=4, \ldots, 10)$ observes at least one individual who receives the highest possible payoff of $a$ by application of strategy $P$. Therefore, at the beginning of Period 2, the first three individuals imitate strategy $R$; all others use strategy $P$. The next diagram in Figure 2 shows a possible realisation of the matching process in Period 2. It can now be verified that, after revision, the first four individuals adopt strategy $R$. The diagrams for the succeeding periods show the spread of strategy $R$ to the entire population by the local imitation dynamic. 
This shows that the state where everyone plays the risk-dominant strategy is also stochastically stable. Hence, limited observability does make an actual difference in the long-run behaviour. $^{7}$

Having established that both absorbing states of the unperturbed process of local imitation may be stochastically stable, we try to examine conditions under which the Pareto-efficient outcome will uniquely prevail in the long-run. The stability of the risk-dominant outcome is clearly driven by the fact that an individual is only partially informed. In the example above, individual 1 only observes individual 2 and this feature may be exploited along the least resistant path of transition from $\vec{P}$ to $\vec{R}$; had individual 1 been able to observe sufficiently many other individuals, he would have been more resistant to this invasion by strategy $R$. This observation, in conjunction with Proposition 3, suggests the next proposition.

Proposition 4. The state where individuals play the strategy of the Pareto-efficient equilibrium is uniquely stochastically stable in all connected networks where each individual observes at least four other individuals.

Proof. By Proposition 3, the state where everyone chooses strategy $P$ is always stochastically stable. Therefore, if we can show that the transition from $\vec{P}$ to $\vec{R}$ takes more than two experimentations when each individual observes at least four other individuals, we can conclude that the state where everyone chooses strategy $R$ is not stochastically stable and this leads to the statement of the proposition.

Let the initial state be $\vec{P}$, i.e. everyone uses strategy $P$. Suppose that two individuals $i$ and $j$ experiment with strategy $R$. Irrespective of the realisation of the random matching process, each individual observes at least one individual with strategy $P$ receiving the highest payoff $a$. To see this, note that there are two types of matches that may be formed after $i$ and $j$ experiment with strategy $R$ :

(a) $i$ and $j$ are matched to each other and so, receive $d$. All other individuals play strategy $P$ and receive $a$; so, none of them switch to strategy $R$. On the other hand, $i$ and $j$ both observe at least one individual receiving the highest payoff $a$ with strategy $P$ and so, they switch to strategy $P$. The state reverts to $\vec{P}$.

(b) $i$ and $j$ are matched to two individuals $k$ and $l$ respectively, who use strategy $P$. Consequently, $i$ and $j$ receive $c$; $k$ and $l$ receive $b$. However, since each individual is linked to at least four other individuals, each of them observes at least one individual who receives the highest payoff $a$ with strategy $P$ (since $M \geq 6$, such an individual exists). So, they choose

\footnotetext{
${ }^{7}$ This contrasts the results derived in Alós-Ferrer and Weidenholzer (2008), where individuals interact with their neighbours but observe and receive information from a wider set of individuals. Individuals choose strategies by imitating the most successful individual they observe. They show that for an appropriately large population, the state where everyone plays $P$ is uniquely stochastically stable. This example may be extended to show that for a population of any size, the state where everyone plays $R$ may also be stochastically stable.
} 
strategy $P$ in the next period. The other individuals in the population continue to choose $P$ as they themselves receive a payoff of $a$ with that strategy. Again, the state reverts to $\vec{P}$.

Thus, the transition from $\vec{P}$ to $\vec{R}$ requires strictly more than two experimentations.

The proposition reveals the need for a minimum amount of connectivity and observability in the network to foist the Pareto-efficient state as the unique stochastically stable state. Further, the condition in the proposition can be interpreted as an absolute condition as it is independent of factors such as population size or the payoff structure. Even though the proposition only captures a sufficient condition, and as such does not provide a full characterization, we now show with an example, that the condition is not very loose.

Example. In Figure 3, we present a network which marginally deviates from the sufficient condition in Proposition 4 and we demonstrate that the transition from $\vec{P}$ to $\vec{R}$ is affected after two initial experimentations, leading to the conclusion that the state $\vec{R}$ is stochastically stable as well. The solid lines in the network indicate the links; only four individuals (marked by the numerals $1,2,3,12$ ) have three links while all other individuals have four neighbours. ${ }^{8}$

The initial state (Period 0 ) is $\vec{P}$. In Period 1 , suppose individuals 1 and 3 experiment with strategy $R$. The realisation of matches is indicated by dotted lines. The triplet next to each individual indicates, from left to right, the current strategy of the individual, the payoff received by the individual as a result of the indicated matching and the strategy that the individual will revise to by local imitation. The diagrams show the transition to $\vec{R}$.

Thus, we see that in face of this small deviation, the transition from $\vec{P}$ to $\vec{R}$ is possible with two experimentations and so, the state $\vec{R}$ is also stochastically stable in this network, enabling the conclusion that the sufficient condition in Proposition 4 is not very loose.

Thus far, no structure is imposed a priori on the shape of the network that governs the flow of information. Now, we turn attention to regular networks. A network is said to be regular when each individual in the population has the same number of links. In terms of our notation, for all individuals $i \in I,|N(i)|=k$ for some positive integer $k \leq M-1$; each individual in the network has $k$ links, where $k$ is the degree of the network.

Proposition 5. For all regular connected networks $N \in \mathcal{N}$, the state where all individuals play the strategy of the Pareto-efficient equilibrium is uniquely stochastically stable.

Proof. To prove the claim made here, it suffices to restrict attention to regular networks of degree two and three. The reason for this is that, firstly, for a population size greater than two, there exists no regular connected network with degree one. Secondly, for regular

\footnotetext{
${ }^{8}$ It is possible to replicate this network to add more individuals such that only four individuals (the first three individuals and the last individual) have three links while others have four links; thus, the fraction of people who have three links goes to zero as the population size increases, and in this sense, this represents a "small" deviation from the condition in Proposition 4.
} 
Period 0.

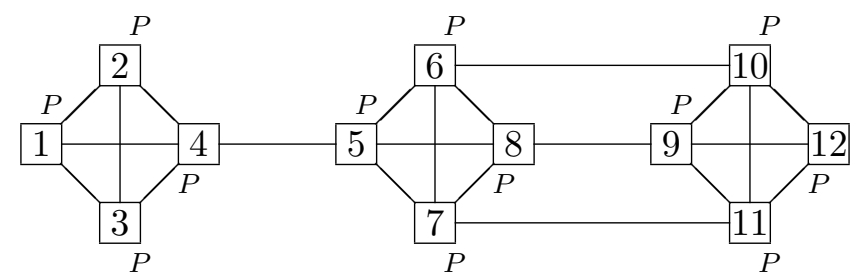

Period 1.

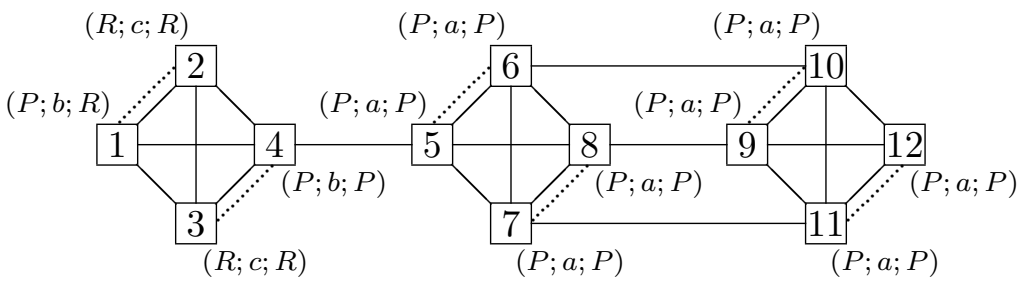

Period 2.

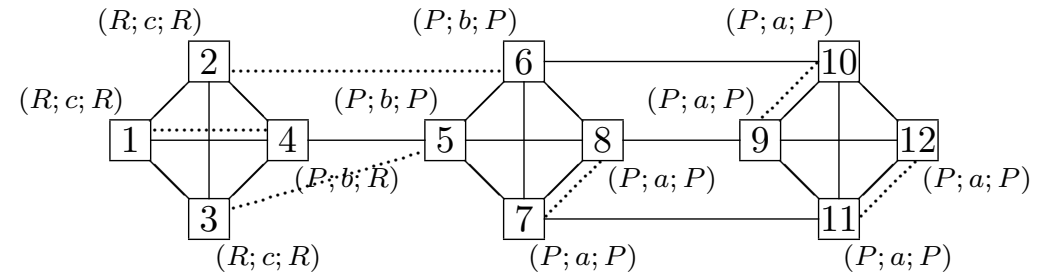

Period 3.

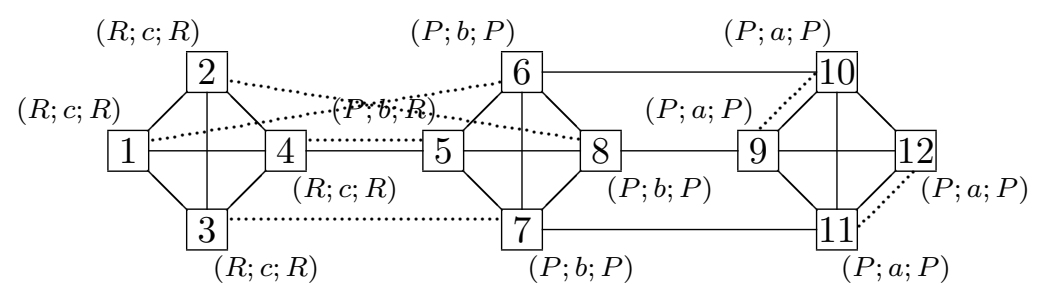

Period 4.

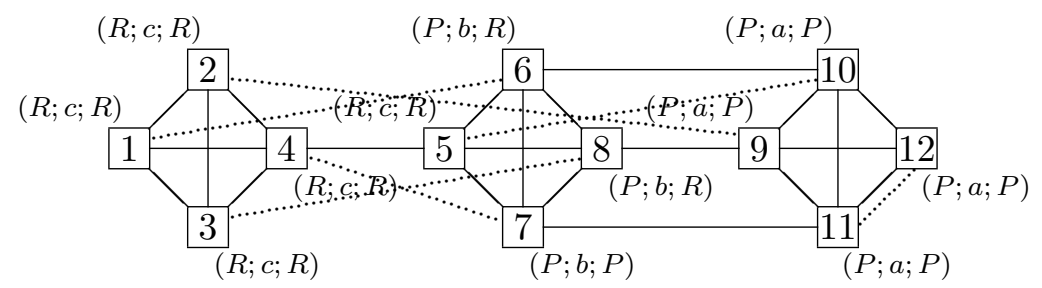

Period 5.

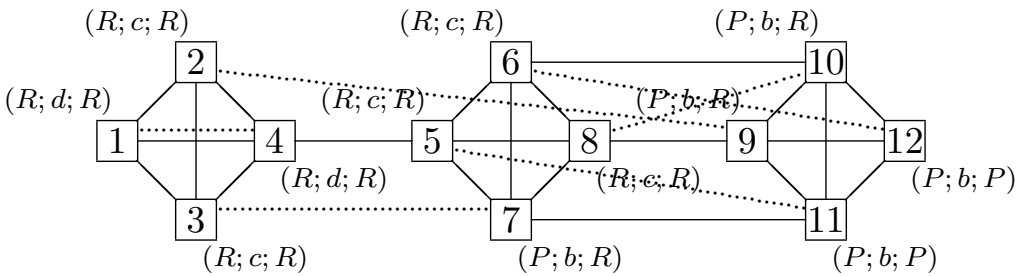

Period 6.

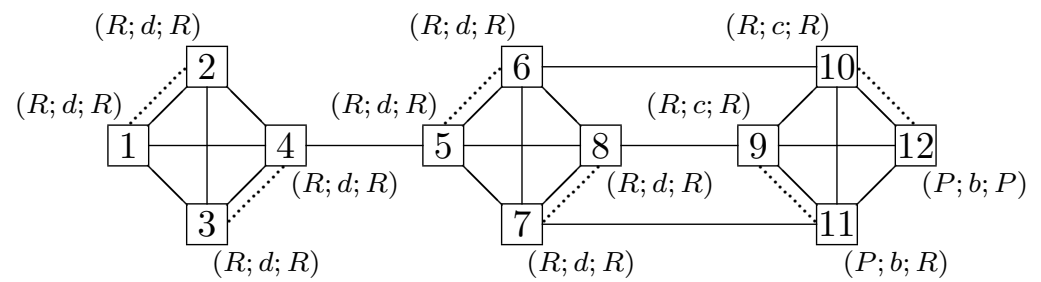

Figure 3: Example to show the sufficient condition in Proposition 4 is reasonably tight. 
networks of degree higher than three, the statement in the proposition may simply be derived from Proposition 4. A short proof is presented here; the appendix contains a more detailed proof.

It will be shown that if two individuals choose $R$ by the way of experimentation, then the number of individuals who play $R$ in the next period (after revision via local imitation) never exceeds two and, hence, cannot spread to the entire population. It can then be concluded that the transition from $\vec{P}$ to $\vec{R}$ requires strictly more than two experimentations, thereby establishing $\vec{P}$ to be the unique stochastically stable state.

Suppose that from the initial state $\vec{P}$, two individuals $i$ and $j$ experiment to strategy $R$. First, also suppose that $i$ and $j$ are matched with each other. Then, they receive a payoff of $d$ while all other individuals receive a payoff of $a$. The resulting payoff profile can be represented by a vector of the form $(d, d, a, \ldots, a)$. Clearly, under the process of local imitation, the individuals who receive $a$ continue to employ strategy $P$. Further, individuals $i$ and $j$ observe two (three) other strategies and payoffs when the regular network has a degree of two (three). Under the assumption of a connected network with at least six individuals, both of them observe strategy $P$ as the more successful strategy and, hence, imitate it. The state reverts to $\vec{P}$.

Now, suppose that $i$ and $j$ are not matched with each other; individual $i(j)$ is matched with $k(l)$. Consequently, individuals $i$ and $j$ receive $c$; individuals $k$ and $l$ receive $b$ while all others receive $a$. The resulting payoff profile can be represented by a vector of the form $(b, b, c, c, a, \ldots, a)$. The individuals who receive $a$ continue to choose strategy $P$; the only individuals who might consider imitating strategy $R$ are $i, j, k$ and $l$. Further, an individual would choose strategy $R$ by imitation only if he does not observe any individual receiving $a$. So, for an individual to choose strategy $R$, it must be that he only observes individuals who did not receive payoff $a$, i.e. for an individual to choose strategy $R$, it must be that he only observes individuals from the set $\{i, j, k, l\}$. We show in the appendix that if more than two individuals only observe individuals from the set, then it violates the condition that we have a connected network of at least six individuals. Hence, no more than two individuals choose strategy $R$; the number of individuals who use this strategy cannot increase.

Again, we comment on the slackness of the sufficient condition above. The example associated with Figure 2, (which was used to demonstrate the stochastic stability of the risk-dominant equilibrium) represents the "smallest" possible deviation from a regular network of degree two for the reason that if one keeps on increasing the number of individuals in the network, then only two individuals continue to share only one $\operatorname{link}^{9}$; thus, the fraction of individuals

\footnotetext{
${ }^{9}$ Further, it is not possible to have a connected network where all but one individual has degree two, which is why the network in Figure 2 represents the smallest possible deviation from a regular connected network of degree two.
} 
who do not have exactly two links goes to zero. Yet, in that network, the state where all individuals play strategy $R$ is stochastically stable. This may permit the conclusion that the sufficient condition is reasonably tight.

\section{Conclusion}

This paper develops a model where individuals belonging to a finite population are engaged recurrently in a strategic environment described by a coordination game where the Paretoefficient equilibrium differs from the risk-dominant equilibrium. The partner in the coordination game is chosen randomly with full support. The payoffs of the individuals depend on the strategy chosen by him and his randomly drawn partner. In addition to his own experience, an individual is, through his (social) links, aware of the experience of his neighbours. An individual is assumed to imitate the strategy of the most successful experience he is aware of. When all individuals are directly linked (the full observability case), the state where everyone chooses the Pareto-efficient equilibrium action is uniquely stochastically stable. However, this result does not fully carry over when individuals only observe a sub-population. The state where everyone plays the Pareto-efficient equilibrium strategy is always stochastically stable but not uniquely so. For uniqueness of this state in the long run, each individual in the network, independent of the population size or payoffs, has to be endowed with a minimum of four links. The Pareto-efficient state is also uniquely stochastically stable for all regular networks.

The sufficient conditions emphasise the importance of connectedness of individuals in a network-structured society. If all individuals are connected to sufficiently large number of individuals, then one would expect the Pareto-efficient outcome to prevail. However, even the mere presence of a small number of loosely connected individuals makes the population vulnerable to being entrenched in a less desirable outcome (i.e. the risk-dominant equilibrium). This hints at the benefit that actions such as community building efforts and other platforms for interaction amongst individuals confer upon the entire population. Encouraging each individual to develop and share links and therefore information results in the population being more immune to invasion of less desirable practises. It is also important to note that the channel via which sufficient amount of observability supports the preferred equilibrium is not peer pressure or monitoring; the purely informational role of observability is enough to nudge the population to the better outcome. 


\section{Appendix}

\section{Proof of Proposition 1.}

We show that from any initial state other than $\vec{P}$ or $\vec{R}$, there is a positive probability of reaching the states $\vec{P}$ or $\vec{R}$. The proof, in three parts, deals with three mutually exclusive situations, where the initial states are such that (i) $0<\omega \leq \frac{M}{2}$, (ii) $\frac{M}{2}<\omega<M$ and even and (iii) $\frac{M}{2}<\omega<M$ and odd, and shows the convergence to either $\vec{P}$ or $\vec{R}$. Before beginning with the proof, we remark on an implication of the network being connected: when the state is such that $0<\omega<M$, there is at least one strategy $P$ individual who is linked to (and so observes) a strategy $R$ individual, and vice-versa (by undirectedness of the links).

Part $I$. When the initial state is such that $0<\omega \leq \frac{M}{2}$, at most half (at least half) of the population plays strategy $P(R)$. In the following period, with positive probability, every strategy $P$ individual is matched to a strategy $R$ individual while the remaining strategy $R$ individuals (if any) are matched amongst each other. All strategy $P$ individuals receive a payoff of $b$ while strategy $R$ individuals receive $c$ or $d$; in either case, all strategy $R$ individuals receive higher payoffs than strategy $P$ individuals. By the observation made in the beginning of the proof, there exists at least one strategy $P$ individual linked to a strategy $R$ individual. For that individual, $R$ represents the more successful strategy and he switches. On the other hand none of the strategy $R$ individuals are induced to change their strategy. Hence, the count of individuals using strategy $R$ increases by at least one.

Again, in the next period, with positive probability, a similar matching may be realised whereby every strategy $P$ individual is matched to a strategy $R$ individual and the remaining strategy $R$ individuals are matched amongst themselves. This continues till the population contains only strategy $R$ individuals. This proves the proposition for all initial states where $0<\omega \leq \frac{M}{2}$.

Part II. Now, the initial states are such that $\frac{M}{2}<\omega<M$ and $\omega$ is even. With positive probability, all strategy $P$ individuals are matched with another strategy $P$ individual. These individuals get the highest payoff of $a$ and so continue to use strategy $P$. This implies that all strategy $R$ individuals are matched with another strategy $R$ individual and they receive a payoff of $d$. Again, there exists at least one strategy $R$ individual who observes a strategy $P$ individual receiving $a$. If the number of such strategy $R$ individuals is even, then the new state is similar in type to the initial state, i.e. $\frac{M}{2}<\omega<M$ and $\omega$ is even, but the number of strategy $P$ individuals is higher; then, we iterate the argument above till the state reaches $\vec{P}$ or the number of strategy $R$ individuals who imitate strategy $P$ is odd. The latter possibility makes the state such that $\omega$ is odd; this case is discussed next.

Part III. Finally, suppose that the initial state is such that $\frac{M}{2}<\omega<M$ and $\omega$ is odd; so, the number of strategy $P$ players is odd. Since the number of individuals in the population is even, this implies that the number of strategy $R$ individuals is also odd. Let the set of 
strategy $P$ individuals who are linked to at least one strategy $R$ individual be denoted by $B$. Let the set of strategy $P$ individuals outside of set $B$ be denoted by $C$; an individual in set $C$ is a strategy $P$ individual who is linked only to other strategy $P$ individuals.

First assume that set $C$ is non-null. With positive probability the following matching process is realised: an individual in set $B$ is matched to another individual in set $B$; if this leaves an individual in set $B$ unmatched (this would happen when the cardinality of this set is odd, and this in particular, subsumes the case where cardinality of set $B$ is one), he is matched with an individual from set $C$ (note the assumption of non-nullity of set $C$ ). Further, the remaining individuals in set $C$ are matched to each other - this might leave an individual in set $C$ unmatched (this would happen when the cardinality of $B$ is odd and set $C$ is non-null), in which case, he is matched to a strategy $R$ individual. This last mentioned strategy $P$ individual gets a payoff of $b$ but is linked to at least another strategy $P$ individual; since all other strategy $P$ individuals receive a payoff of $a$, he imitates strategy $P$. Thus, following this realisation of random matching, all individuals in set $B \cup C$ observe strategy $P$ as the more successful strategy and choose it in the succeeding period. Further, the strategy $R$ individuals linked to strategy $P$ individuals imitate strategy $P$. Hence in the new state, the number of strategy $P$ individuals increase; this argument is iterated till the population is solely comprised of strategy $P$ individuals.

Now, suppose that set $C$ is null i.e. each of the strategy $P$ individuals (who are odd in number) are linked to at least one strategy $R$ individual and hence are contained entirely in set $B$. First suppose that there is one individual (say, $i$ ) in set $B$, who apart from being linked to a strategy $R$ individual, is also linked to another strategy $P$ individual (say, $j$ ). ${ }^{10}$ In case of the event that there is more than one such individual, let the individual with the least number of links to strategy $R$ individuals be designated as individual $i$ (in case of a tie, the choice of individual $i$ can be made randomly). Then, with positive probability, individual $i$ is matched to a strategy $R$ individual whereas individuals in $B \backslash\{i\}$ (the cardinality of which is even) are matched amongst themselves. Following this, all individuals in set $B$ continue to employ strategy $P$ as: (i) all individuals in that set except individual $i$ receive the highest payoff and (ii) individual $i$ observes individual $j$ doing so. On the other hand, the strategy $R$ individuals linked to individuals in $B \backslash\{i\}$ (by construction of set $B$ and individual $i$, there is at least one such strategy $R$ individual) imitate strategy $P$. Again, the number of strategy $P$ individuals increase, and the arguments used thus far can be iterated till we reach the state $\vec{P}$.

Finally suppose that in addition to set $C$ being null, none of the individuals in set $B$ are connected amongst themselves. Then, since the initial state is described by $\omega>\frac{M}{2}$ (i.e. since

\footnotetext{
${ }^{10}$ The situation where set $C$ is null and there is no individual in set $B$ who is linked to another individual in set $B$ is discussed in the next paragraph.
} 
strategy $P$ individuals outnumber strategy $R$ individuals and since no strategy $P$ individual is connected to another strategy $P$ individual), there must exist at least one strategy $R$ individual, say $k$, who is connected to more than one strategy $P$ individual. So, assume that $k$ is linked to at least two strategy $P$ individuals, say $u$ and $v$. With positive probability, all individuals in set $B \backslash\{u\}$ (the cardinality of which is even) are matched amongst themselves and so, receive the highest payoff and continue to play strategy $P$. Individual $u$ is then matched with a strategy $R$ individual and receives a payoff of $b$ and observes other strategy $R$ individuals receiving either $d$ or $c$, each of which is larger than $b$; consequently, he revises to strategy $R$. At the same time, individual $k$ switches from strategy $R$ to $P$ on observing individual $v$ 's success with strategy $P$. (If any strategy $R$ individual is connected to an individual in $B \backslash\{u\}$, he switches to $P$ by imitation.) Thus, in the new state, in no case does the number of strategy $P$ individuals decrease; but now, in this new state, we are back to the situation where at least one strategy $P$ player (individual $k$ ) is linked to another strategy $P$ individual (individual $v$ ). So, we have a situation where $\omega>\frac{M}{2}$ and an individual in set $B$ is connected to another individual in set $B$. Hence, we can use the argument in the preceding paragraph to argue that, with positive probability, the number of strategy $P$ individuals increases, and the arguments used thus far can be iterated till we reach the state $\vec{P}$.

Hence, we have demonstrated that whenever the state is such that $\omega>\frac{M}{2}\left(\omega \leq \frac{M}{2}\right)$, with positive probability the number of strategy $P(R)$ individuals increases and, therefore, there exists a path to the state $\vec{P}(\vec{R})$. This establishes the proposition.

\section{Proof of Proposition 3.}

The proof is in two steps. Part I shows that the $\rho(\vec{R} \rightarrow \vec{P})=2$. Part II demonstrates that $\rho(\vec{P} \rightarrow \vec{R}) \geq 2$. This establishes the result as $\rho(\vec{R} \rightarrow \vec{P}) \leq \rho(\vec{P} \rightarrow \vec{R})$.

Part $I$. Let the initial state be $\vec{R}$, i.e. everyone uses the risk-dominant equilibrium strategy $R$. Suppose two individuals $i$ and $j$ - to simplify matters, assume that they are neighbours experiment with strategy $P$. With positive probability, they are also matched to each other and they realise the highest payoff of $a$. This is observed by the neighbours of $i$ and $j$. Consequently, the individuals who use strategy $P$ in the next period are $N(i) \cup N(j)$ (note that individuals $i$ and $j$ are included in this set). Suppose that this does not make up the entire population. Then there are two possibilities corresponding to the cardinality of the set $N(i) \cup N(j)$ being odd or even. If it is odd, then with positive probability, individuals in the set $N(i) \cup N(j) \backslash\{i\}$ (the cardinality of this set is even) find their match in the same set, while individual $i$ is matched with a strategy $R$ individual. As individuals in $N(i) \cup N(j) \backslash\{i\}$ themselves realise the highest payoff, they continue to use strategy $P$ while individual $i$ does so because he observes individual $j$. But now, in addition, the neighbours of individuals in $N(i) \cup N(j)$ also imitate this strategy. Hence, the set of individuals who now use strategy $P$ expands to $N^{2}(i) \cup N^{2}(j)$. 
On the other hand, if the cardinality of the set $N(i) \cup N(j)$ is even, then with positive probability, each individual in this set is matched to another individual from this set. They receive the highest payoff and their strategy is imitated by their neighbours. Again, the the set of individuals who now use strategy $P$ expands to $N^{2}(i) \cup N^{2}(j)$. Now, the same reasoning can be used to conclude that the set of individuals who use strategy $P$ expands to $N^{K}(i) \cup N^{K}(j)=I$ (where $K$ is the farthest distance between two individuals in the population): if for some $k \geq 2$, the cardinality of $N^{k}(i) \cup N^{k}(j)$ is even, then with positive probability, all individuals in that set are matched to another individual from the set; if the cardinality is odd, then all individuals except individual $i$ are matched to another individual from the set. In any case, by local imitation, the strategy spreads to $N^{k+1}(i) \cup N^{k+1}(j)$ until it encompasses the entire population. This shows $\rho(\vec{R} \rightarrow \vec{P}) \leq 2$, but it is trivial to see that $\rho(\vec{R} \rightarrow \vec{P})>1 ;$ so, $\rho(\vec{R} \rightarrow \vec{P})=2$.

Part II. Suppose that from initial state $\vec{P}$, where everyone uses strategy $P$, individual $i$ experiments to strategy $R$. This individual is matched to a strategy $P$ individual, $j$. Individual $i(j)$ receives a payoff of $c(b)$. By the assumption that the population consists of at least six individuals and by connectedness of the network, it must be true that $i$ or $j$ (if not both) observe a strategy $P$ individual receiving a payoff of $a$, and so chooses strategy $P$ in the next period. Then, in the next period, at most one individual still chooses strategy $R$. Thus, it is not possible for strategy $R$ to grow in the population, leading to the conclusion that the transition from $\vec{P}$ to $\vec{R}$ requires at least two experimentations.

\section{Proof of Proposition 5.}

It will be argued that when two individuals experiment with strategy $R$ when the initial state is $\vec{P}$, then the number of individuals who choose strategy $R$ in the succeeding periods will never exceed two; hence, the transition from $\vec{P}$ to $\vec{R}$ is not tenable with two experimentations. This proves that $\vec{P}$ is the only stochastically stable state.

Assume that two individuals $i$ and $j$ experiment with strategy $R$. If they are matched to each other, then they receive a payoff of $d$ while everyone else receives $a$. Hence, the only individuals who might consider playing strategy $R$ in the succeeding period are $i$ and $j$. However, since they are linked to two or three other individuals (depending on the degree of the network), they always observe another individual receiving a payoff of $a$ and imitate the action of choosing strategy $P$. It follows that if there is any chance for the population to transit to $\vec{R}$ after the two individuals experiment with strategy $R$, then they must be matched with a strategy $P$ individual and not with themselves. Resultantly, the focus is on situation where individuals $i$ and $j$ are matched to two strategy $P$ individuals, $k$ and $l$ respectively. Now, the only individuals who might consider imitating the use of strategy $R$ are $i, j, k, l$ as all others end up receiving the highest payoff $a$ (recall that we deal with populations containing at least six individuals). It will be shown that in the next period, of these four individuals, no 
more than two individuals will consider using strategy $R$ and hence the use of this strategy cannot spread to the entire population. Part I shows this for regular networks of degree two while Part II does the same for regular networks of degree three.

Part I. An individual belonging to the set $D=\{i, j, k, l\}$ will choose strategy $R$ by imitation only if he observes strategy $R$ as the most successful strategy in his neighbourhood. This in turn happens only if he shares both his links with two other individuals from the set $D$. Otherwise, he is linked to a strategy $P$ individual who receives the payoff of $a$ and imitates strategy $P$. So, let us suppose that two individuals, without loss of generality, $i$ and $j$ have both their neighbours in set $D$, thereby implying that both of them choose strategy $R$ in the next period. It will be seen that, given this, it is not possible for either $k$ or $l$ to share both their links with individuals from the set $D$. Hence, both $k$ and $l$ observe at least one individual outside the set $D$ and they imitate strategy $P$, thereby making the spread of strategy $R$ impossible.

A connected regular network of degree two can only be a Hamiltonian cycle. Further, in order for both individuals $i$ and $j$ to have two neighbours from set $D$, it must be that they observe each other, while the other neighbour of individual $i(j)$ is $k(l)$. If the network has at least six individuals, then it follows that individuals $k$ and $l$ must have a neighbour who receives the highest payoff from the use of strategy $P$. So, individuals $k$ and $l$ imitate strategy $P$, and in the next period, no more than two individuals use strategy $R$. The following paragraphs demonstrate this in more detail.

First, assume that $i$ and $j$ do not observe each other, i.e. $j \notin N(i)$ and $i \notin N(j)$. Then it must be that $N(i)=N(j)=\{k, l\}$ (by the assumption that $i$ and $j$ observe two individuals from the set $\{i, j, k, l\})$. But then this means that $N(k)=N(l)=\{i, j\}$ - a contradiction to the assumption that the regular network of degree two consists of at least six individuals and is connected. (Note that it is not possible to include a fifth individual as $i, j, k, l$ already have exhausted both their links.)

So, let us assume that $i$ and $j$ do observe each other, i.e. $j \in N(i)$ and $i \in N(j)$. Then there are two possible cases:

(i) $i$ and $j$ observe a common individual from the set $D$, say $k$. So, $N(i)=\{j, k\}$, $N(j)=\{i, k\}$ and $N(k)=\{i, j\}-$ a contradiction to the assumption that the regular network of degree two consists of at least six individuals, and is connected. (Note that it is not possible to include a fourth individual as $i, j, k$ already have exhausted both their links.)

(ii) $i$ and $j$ observe different individuals from the set $D$, say $k$ and $l$ respectively. So, $N(i)=\{j, k\}, N(j)=\{i, l\}, i \in N(k)$ and $j \in N(l)$. As mentioned earlier, in order for individuals $k$ and $l$ to choose strategy $R$ in the next period, it must be that they observe two individuals from the set $D$. The only such possibility is when $N(k)=\{i, l\}$ and $N(l)=\{j, k\}$ - but again, this contradicts the assumption that the regular network of degree two consists 
of at least six individuals.

This proves that the use of strategy $R$ does not spread beyond two individuals in the population in a regular network of degree two that contains at least six individuals and so, the transition from $\vec{P}$ to $\vec{R}$ needs more than two experimentations.

Part II. Now, each individual observes three other individuals. For an individual in $D=\{i, j, k, l\}$ to choose strategy $R$ by local imitation after the initial experimentation by $i$ and $j$, it has to be that he observes the other three individuals in this set. Again, assume that two individuals, say $i$ and $j$ observe three individuals from the above set; in this event, it will be seen that it is not possible for $k$ and $l$ to choose strategy $R$, thereby limiting the use of this strategy to two individuals (at most). By assumption, $N(i)=\{j, k, l\}$ and $N(j)=\{i, k, l\}$; so, $\{i, j\} \subset N(k)$ and $\{i, j\} \subset N(l)$. If $k$ is to choose strategy $R$ by imitation, then, it must be (by undirectedness of links) that $N(k)=\{i, j, l\}$ and therefore, $N(l)=\{i, j, k\}$. But then this contradicts the assumption that the regular network of degree three has at least six individuals, and is connected. Hence, it is not possible for more than two individuals to

choose strategy $R$ by local imitation. This again proves that the transition from $\vec{P}$ to $\vec{R}$ needs more than two experimentations.

\section{References}

1. C. Alós-Ferrer and S. Weidenholzer (2008). Contagion and Efficiency. Journal of Economic Theory 143 (1): 251-274.

2. J. Apesteguia, S. Huck and J. Oechssler (2007). Imitation: Theory and Experimental Evidence. Journal of Economic Theory 136 (1): 217-235.

3. A. Banerjee and D. Fudenberg (2004). Word of Mouth Learning. Games and Economic Behavior 46 (1): 1-22.

4. K. Basu and J. Weibull (2003). Punctuality - A Cultural Trait as an Equilibrium. In: R. Arnott et al. (Eds.), Economics for an Imperfect World: Essays in Honor of Joseph Stiglitz, MIT Press.

5. J. Björnerstedt and J. Weibull (1996). Nash Equilibrium and Evolution by Imitation. In: K. Arrow, E. Colombatto, M. Perlman, C. Schimdt (Eds.) The Rational Foundations of Economic Behaviour. Macmillan Press.

6. R. Dawkins (1976). Selfish Gene. Oxford University Press.

7. G. Ellison and D. Fudenberg (1993). Rules of Thumb for Social Learning. Journal of Political Economy 101 (4): 612-643. 
8. G. Ellison and D. Fudenberg (1995). Word-of-Mouth Communication and Social Learning. The Quarterly Journal of Economics 110 (1): 93-125.

9. S. Goyal and F. Vega-Redondo (2005). Network Formation and Social Learning. Games and Economic Behaviour 50 (2): 178-207.

10. M. Kandori, G. Mailath and R. Rob (1993). Learning, Mutation and Long-Run Equilibria in Games. Econometrica 61 (1): 29-56.

11. A. Robson and F. Vega-Redondo (1996). Efficient Equilibrium Selection in Evolutionary Games with Random Matching. Journal of Economic Theory 70 (1): 65-92.

12. E. Ullman-Margalit (1978). Invisible Hand Explanations. Synthese 39: 263-291.

13. H.P. Young (1993). The Evolution of Conventions. Econometrica 61 (1): 57-84.

14. H.P. Young (1998). Individual Strategy and Social Conventions. Princeton University Press. 